\title{
IV.3 Die Beilegung des Saarproblems 1955-1957
}

\section{IV.3.1 „Nein“ zum Saarstatut: Das Referendum vom 23, 10. 1955}

\section{„Latente Hypothek auf den deutsch-französischen Beziehungen“}

Die großen Hoffnungen Frankreichs hinsichtlich der Saar waren in den Jahren nach 1945 auf die Forderung nach einer Loslösung von Deutschland und der Umwandlung in ein politisch autonomes Gebiet zusammengeschmolzen. Mit dem Abkommen vom 23. 10. 1954 hatten sich Bonn und Paris auf die „Europäisierung" des Territoriums unter dem Dach der Westeuropäischen Union und auf eine Volksabstimmung verständigt, in der die Bevölkerung das Statut nur akzeptieren oder ablehnen durfte, ein Votum für eine Angliederung an die Bundesrepublik aber ausgeschlossen war. Ein halbes Jahr später, Ende April 1955, vereinbarten beide Regierungen, den Rat der WEU bei seiner konstituierenden Sitzung um Übernahme der ihm überantworteten Verpflichtungen zu bitten. Zugleich einigten sich die Außenminister Adenauer und Pinay darauf, gemeinsame Empfehlungen über die Anwendung des Saarabkommens, die Durchführung der Volksabstimmung, die Zusammensetzung der Kontrollkommission sowie die Befugnisse des Kommissars vorzulegen. Details derartiger Vorschläge klammerten sie bewußt aus, um den Abschluß des noch laufenden Ratifikationsverfahrens nicht durch etwaige Differenzen zu gefährden ${ }^{1}$.

Damit war aber quasi vorprogrammiert, daß die Saarfrage auch nach dem Inkrafttreten der Pariser Verträge im Mai nicht zur Ruhe kommen, sondern vorerst weiter als "latente Hypothek auf den deutsch-französischen Beziehungen“ lasten würde ${ }^{2}$. Denn beiderseits des Rheins begann nun das Tauziehen um eine Optimierung der Ausgangsbedingungen für das bevorstehende Referendum. Konkret ging es um die Einrichtung einer WEU-Kommission zur Überwachung des Verfahrens, um den Kreis der Abstimmungsberechtigten und die Befugnisse des künftigen Saarkommissars. Beiden Regierungen blies dabei der Wind innenpolitisch kalt ins Gesicht. Während sich Adenauer in der Bundestagsdebatte vom 6.5. heftiger Vorwürfe von seiten der FDP an seiner Saarpolitik ausgesetzt sah"3 ${ }^{3}$ erntete Pinay starke Kritik dafür, daß er Ende April eine Stärkung der Position des Kommissars bei Verstößen der Saarregierung gegen das Saarstatut akzeptiert hatte. Der von französischen Parlamentariern wie der saarländischen Regierung ausgehende Druck ${ }^{4}$ führte umgehend zu einer Versteifung in der Haltung des Quai d'Orsay. Die Adenauer am 7.5. von Justizminister Schuman präsentierten neuen Vorschläge über die Kompetenzen des Kommissars wichen von den bisherigen Vereinbarungen insofern ab, als sie nur noch dem WEU-Rat das Recht zubilligten, in schwerwiegenden Fällen statutwidrige Maßnahmen von Saarorganen auszusetzen. Für den Bundeskanzler war dies ebenso inakzeptabel wie die Empfehlungen des Justizministers zum Abstimmungsrecht der aus dem Saarland Ausgewiese-

1 Vgl. Kap. III.3.4.

2 PA, Abg.-Liste Ref. 204, Bd. 6, von Maltzan an AA, 205-00 I 3669/55, 4. 8. 1955.

3 S. Bundestagsdebatte, 6. 5. 1955, in: BT, Sten. Ber., Bd. 24, S. 4462-4480.

4 Vgl. Schmidt, Saarpolitik, Bd. 3, S. 162 f;; Thoß, Lösung, S. 274. 
nen 5 . Eindringlich wies er Pinay einen Tag später auf die ihn erwartenden Schwierigkeiten, wenn die alten Abmachungen, die er gegenüber dem Kabinett und dem Auswärtigen Ausschuß des Bundestages bereits bekanntgegeben hatte, nun nachträgliche Abänderungen erführen. Die Widerstände in Paris und Saarbrücken wirkten auf ihn um so unverständlicher, als er fest davon ausging, daß das Referendum mit einer Dreiviertel-Mehrheit angenommen würde. Worauf es seiner Meinung nach ankam war, für den Posten des Kommissars einen Mann zu finden, der durch seine Autorität und die ihm zugestandenen Rechte Reibungen verhinderte. Der französische Außenminister zeigte sich zwar aufgeschlossen gegenüber den Sorgen des Kanzlers, wies aber unmißverständlich auf die Drohung des saarländischen Ministerpräsidenten, das Referendum zu verhindern, wenn sein Kabinett zum „Sklaven des Saarkommissars“ würde. Pinay sprach sich daher dafür aus, dem WEU-Rat die Entscheidung zu überlassen ${ }^{6}$.

Adenauer hegte aus politischen wie psychologischen Erwägungen Bedenken, diesem Wunsch zu entsprechen. Nachdem er noch am selben Tag telefonisch die englische Regierung darum gebeten hatte, sich für einen starken Saar-Kommissar in der WEU einzusetzen ${ }^{7}$, vertrat er in einem Schreiben an den französischen Außenminister am 9.5. plötzlich die Ansicht, es käme gar nicht zu dringend nach einem Beschluß verlangenden Verletzungen des Saarstatuts. Er bäte daher, der Saarregierung mitzuteilen, „daß aus rechtlichen und psychologischen Gründen es zweckmäßig sei, die Angelegenheit nicht weiter zu verfolgen"8. Pinay konnte dem Gesuch angesichts der Widerstände aus Saarbrücken nicht zustimmen. Adenauer hingegen scherte sich nicht um die Kritik Hoffmanns und dessen "leere Drohungen“. "Denken Sie“, so redete er ihm in einer neuerlichen Begegnung am 10.5. ins Gewissen, „doch an unser Ziel: Ruhe zu schaffen und deutsch-französische Schwierigkeiten zu vermeiden. Das Einverständnis der Saarregierung brauchen wir nicht." Aufgrund der Hartnäckigkeit des Außenministers blieb dem Kanzler aber nichts anderes übrig, als darin einzuwilligen, den WEU-Rat über die Befugnisse des Kommissars und den Kreis der Abstimmungsberechtigten beim Referendum urteilen zu lassen9. Mit dieser Übereinkunft ließen beide Politiker allerdings die Kritiker in Bonn und Paris nicht verstummen ${ }^{10}$.

Als der WEU-Rat und sein turnusmäßiger Vorsitzender Macmillan sich in der konstituierenden Sitzung am 11.5. des Problems annahmen, wurde rasch deutlich, daß ihnen die saarländischen Forderungen weder gerechtfertigt noch demokratisch erschienen ${ }^{11}$. Und so beschlossen sie in der Frage der Abstimmungsberechtigten, unter Berücksichtigung der Hinweise Adenauers auf den Vorbild-

5 S. Unterredung zwischen Adenauer und Schuman vom 7. 5. 1955, in: DDF 1955, Bd. 1, S. 600 f.; AMAE, Europe 1944-1960, Sarre, Bd. 274, Bl. 71-73, Hallstein an Massigli, 8. 5. 1955.

- BA, NL Blankenhorn, Bd. 41ba, Bl. 102-104, Unterredung zwischen Adenauer und Pinay vom 8. 5. 1955, Streng geheim.

7 Vgl. Thoß, Lösung, S. $274 \mathrm{f}$.

8 Adenauer an Pinay, 9. 5. 1955, in: Adenauer, Briefe 1953-1955, S. 279f., hier S. 280.

9 BA, NL Blankenhorn, Bd. 41ba, Bl. 89-94, Unterredung zwischen dens. vom 10.5. 1955, Streng geheim.

to S. Artikel Hück, in: „Die Rheinpfalz“, 10. 5. 1955, im Auszug auch in: BDFD III, S. 499 f.

11 S. Pinay an Grandval, Tel. 313/17, 23. 5. 1955, Réservé, in: DDF 1955, Bd. 1, S. $676 \mathrm{f}$. 
charakter für etwaige Volksbefragungen in Ostdeutschland ${ }^{12}$ auch diejenigen zum Plebiszit zuzulassen, die wegen ihrer prodeutschen Einstellung nach 1945 von der französischen Besatzungsmacht ausgewiesen worden waren. Nur in begründeten Einzelfällen durfte Saarbrücken Einspruch gegen die Rückkehr erheben, der dann von der WEU-Überwachungskommission überprüft werden sollte. Hinsichtlich der Befugnisse des Kommissars billigten sie dem Amtsinhaber in "dringenden und schwerwiegenden Fällen" das Recht zu, Maßnahmen der Saarregierung bis zu einer definitiven Entscheidung der WEU für wenige Tage auszusetzen ${ }^{13}$. Auf dieser Basis verabschiedete der Rat zwei weitere Entschließungen, mit denen er die Bestimmungen des Saarstatuts billigte und eine Kommission zur Überwachung des Referendums einsetzte ${ }^{14}$.

Desillusioniert und mit großer Zukunftsangst nahm Hoffmann die Kompromißlinie zwei Tage später $a^{15}$. Seine Sorgenfalten vertieften sich noch, als es ihm nicht gelang, die erst nach dem Referendum vorgesehene Ernennung des WEUKommissars vorzuziehen. Die WEU widersetzte sich dem Wunsch und beschloß, einen Vertreter zu ernennen, dessen Wahl bis zum Referendum provisorisch sein sollte ${ }^{16}$. Hoffmann, der nach den Abfuhren der WEU mit dem Gedanken spielte, einen Antrag auf Mitgliedschaft zu stellen ${ }^{17}$, wünschte sich auf diesem Posten van Naters ${ }^{18}$. In Paris war ursprünglich daran gedacht, einen Engländer mit dem Amt des Saarkommissars zu betrauen. Unter dem Druck der Schwerindustrie nahm die Regierung davon wieder Abstand und favorisierte die Kandidatur eines Schweizers oder einer Persönlichkeit aus den BENELUX-Staaten. Die Wahl von van Naters schloß man nicht aus. Für die Bundesregierung kam eine Ernennung des holländischen Europarat-Abgeordneten aber nicht in Frage ${ }^{19}$. Ohne seine persönliche Integrität anzweifeln zu wollen, galt er als Repräsentant eines im Jahr zuvor gescheiterten Lösungsversuchs zur Saarfrage. Statt dessen hielt Bonn daran fest, einen Engländer mit dem Amt zu betrauen. Da die Briten aber auf diesen Wunsch nur nach einer deutsch-französischen Verständigung eingehen wollten, mußte zunächst der Kontakt zu Paris gesucht werden ${ }^{20}$.

Aus Unterredungen im Quai d'Orsay gewann der deutsche Botschafter von Maltzan Ende Juni den Eindruck, als sei die Kandidatur von van Naters „endgültig erledigt“. Pinay, so berichtete er ins Auswärtige Amt, habe zugesagt, eine Kandidatenliste aufzustellen und mit Adenauer zu besprechen ${ }^{21}$. Dennoch entfachten christdemokratische bzw. liberale deutsche Presseorgane einen Feldzug

12 Vgl. Erklärung Adenauers in der Sitzung des CDU-Bundesvorstandes, 3.6. 1955, in: Buchstab (Bearb.), Protokolle 1953-1957, S. 502 f.

13 Entschließung des WEU-Rats, 11.5. 1955, in: EA 1955, S. 7924.

14 S. Entschließungen dess., 11.5. 1955, in: ebd., S. 7924-7926; Pinay an Chauvel, Tel. 6598/603, 12. 5. 1955, Très secret, Réservé, in: DDF 1955, Bd. 1, S. 622f.; Thoß, Lösung, S. 275.

15 S. Grandval an Pinay, Tel. 396/400, 16. 5. 1955, in: DDF 1955, Bd. 1, S. 657-659; Hoffmann, Ziel, S. $402-405$.

16 S. Hoffmann an Macmillan, 22. 6. 1955 (Übersetzung), in: DDF 1955, Bd. 1, S. 820.

17 S. Grandval an Pinay, Tel. 428/31, 1.6. 1955, Réservé, in: ebd., S. 707 f.

I8 S. AMAE, Europe 1944-1960, Sarre, Bd. 274, Bl. 229-230, ders. an MAE, Tel. 410/15, 25. 5. 1955, Réservé.

19 S. PA, B 17, Bd. 23, Bl. 81, von Maltzan an AA, FS Nr. 378, 24. 6. 1955, Verschlüsselt.

20 S. ebd., Bl. 74, Hallstein an London etc., Tel. AZ St.S. 746/55, 3. 6. 1955.

21 Ebd., Bl. 102, von Maltzan an AA, FS Nr. 389, 30. 6. 1955, Verschlüsselt. 
gegen van Naters, dem vorgeworfen wurde, der Saarbrücker Regierung zu nahe zu stehen. In der französischen Diplomatie entstand dadurch der Eindruck, als ob das Bundeskanzleramt den Holländer definitiv zum Rückzug bewegen wolle22. Vor diesem Hintergrund lehnte der WEU-Rat auf seiner Sitzung am 30.6. den saarländischen Wunsch nach einer vorgezogenen Bestallung des Kommissars gänzlich $a b^{23}$.

Kurz zuvor hatte der Rat Kenntnis von der Einsetzung der neutralen Überwachungskommission durch die Regierungen der nicht direkt beteiligten Staaten genommen. Am 1. 7. nahm das fünfköpfige Gremium seine Arbeit unter dem Vorsitz des belgischen Senators Fernand Dehousse auf24. Als Verbindungsmann fungierten für die Bundesrepublik Hans-Albrecht Schwarz-Liebermann aus dem Auswärtigen Amt, für Frankreich der neue Botschafter in Saarbrücken, Eric de Carbonnel, ein im Gegensatz zu seinem Vorgänger eher bescheidener, nüchterner und aufrichtiger Diplomat ${ }^{25}$. Grandval, der mit Hoffmann in den prodeutschen Kreisen an der Saar seit Jahren zu den „bestgehaßten Männern“ 26 zählte, mußte zum 30. 6. seine Stellung aufgeben und wurde als Generalresident nach Marokko geschickt. Wiederholt hatte er in den letzten Wochen seine Regierung ermahnt, die Stimmung des saarländischen Partners, bei dem die Überzeugung wuchs, als Instrument benutzt zu werden, stärker zu berücksichtigen ${ }^{27}$. Seine Klagen mochte man aber in Paris nicht mehr hören. Sie wirkten angesichts der prioritären Pflege der deutsch-französischen Beziehungen politisch deplaziert, zumal Adenauer offenbar darum gebeten hatte, den reibungslosen Verlauf des Referendums nicht durch die Präsenz Grandvals zu stören ${ }^{28}$. Faure und Pinay gingen darauf ein, weil sie den selbstbewußten „Prokonsul“ 29 ohnehin als unbequem empfanden, da er auf eine Kontinuität der französischen Saarpolitik pochte, die sie nicht mehr zu halten bereit waren. Nachdem sie ihm zunächst signalisiert hatten, er möge für die Zeit des Abstimmungskampfes Urlaub nehmen, lobten sie ihn schließlich auf den Posten nach Marokko weg30.

Der Abgang des ihr seit Jahren vertrauten Ansprechpartners war für der Saarregierung um so schwerer zu verdauen, als sie sich jetzt der Herausforderung gegenüber sah, Gesetze zu erlassen, die in der dreimonatigen Vorbereitungszeit der Volksabstimmung die politischen Freiheiten zu gewährleisten hatten. Im einzelnen betraf dies die Zulassung der bisher unterdrückten Parteien, die Genehmigung von Parteineugründungen und die Billigung der Presse- und Versammlungsfreiheit. Für Hoffmann bedeutete dies einen schwierigen Spagat. Denn einerseits konnte er kein Interesse daran haben, der Opposition Freiräume zu bieten, die es

22 S. de Margerie an Pinay, Tel. 2563/65, 1. 7. 1955, in: DDF 1955, Bd. 2, S. 1 f.

23 Vgl. ebd., Bd. 1, S. 820, Anm. 2.

24 S. Chauvel an Pinay, Tel. 3093/100, 16.6. 1955, in: ebd., S. 783-785; Schmidt, Saarpolitik, Bd. 3, S. $165 \mathrm{f}$. Weitere Mitglieder waren der englische Diplomat Rendel, der italienische Diplomat de Paolis, der niederländische Gesandte Bentinck und der luxemburgische Minister Kunnen.

${ }_{25}$ S. Lahr an seine Mutter, 24. 10. 1955, in: Lahr, Zeuge, S. 238 f.; Schmidt, Saarpolitik, Bd. 3, S. 167.

26 Schneider, Gilbert Grandval, S. 240.

27 S. Grandval an Pinay, Tel. 396/400, 16. 5. 1955, in: DDF 1955, Bd. 1, S. 657-659; ders. an dens., Tel. 428/31, 1. 6. 1955, Réservé, in: ebd., S. 707 f.; Schneider, Gilbert Grandval, S. 239.

28 Vgl. ebd., S. 240.

29 Ebd., S. 201.

30 Vgl. Freymond, Saar, S. 195; Schneider, Gilbert Grandval, S. 240 f. 
ihr erlaubten, die Bevölkerung gegen das Saarstatut aufzustacheln. Andererseits drohte bei einem zuwenig an politischen Freiheiten ein Konflikt mit der Bundesregierung, die die neue Gesetzgebung als wichtigen Anhaltspunkt für die eigene Saarpolitik der nächsten Monate begriff. Sollte sie am Saarstatut festhalten oder auf ein Nein im Referendum setzen?

Entgegen der Auffassung Adenauers, daß die überwältigende Mehrheit der Saarländer mit Ja stimmen werde, hatten demoskopische Umfragen eine breite Unentschlossenheit der Saarländer indiziert: Nur 21\% der Befragten sprachen sich im April für, 20\% gegen das Statut aus, aber 59\% schwankten noch ${ }^{31}$. Ende Juni meldete der Leiter des Saarreferats im Auswärtigen Amt, Thierfelder, dem neuen Außenminister von Brentano einen zunehmenden Anstieg bei den NeinSagern und beklagte sich darüber, wie schwer es werde, die Ja-Parole in der CDUSaar durchzusetzen. Die Entwicklung in der Meinungsbildung warf für die Bundesregierung vor allem deshalb so große Probleme auf, weil die bisher bekannten Referentenwürfe zu den saarländischen Gesetzen, die Hoffmann bereits am 9.7. in Kraft treten zu lassen gedachte, „starke polizeistaatliche Züge“ aufwiesen. Die Bonner Reaktion konnte vor dem Hintergrund der schon einsetzenden heftigen Kritik der Parteien kaum positiv ausfallen. Da man sich aber nicht in einen Gegensatz zur Dehousse-Kommission setzten wollte, schickte von Brentano kurzerhand Schwarz-Liebermann zu Dehousse, um seine Position klarzumachen und einen Gesprächstermin zu erbitten ${ }^{32}$.

Nachdem der Senator zugesagt hatte, sich dafür einzusetzen, daß die Oppositionsparteien an der Saar ausgiebig Gelegenheit zur Stellungnahme über die politischen Freiheiten vor der Kommission bekämen ${ }^{33}$, hielt die in der deutschen Presse geführte Kampagne einen Augenblick inne. Sie wurde aufs neue entfacht, als der saarländische Landtag am 8. 7. die vier Gesetze für das Referendum verabschiedete ${ }^{34}$. Sie richtete sich nicht nur gegen die Dokumente, sondern auch gegen Dehousse. Zusätzliches Öl ins auflodernde Feuer gossen deutsch-kritische Zeitungen, die enthüllten, wie die „officines du ministre Jacob Kaiser“ mit Hilfe des Bundespresseamtes heimlich Propagandamaterial ins Saargebiet einschmuggel$\operatorname{ten}^{35}$.

Als Vorbedingung zur Billigung der vier Gesetze forderte die Kontrollkommission der WEU von der saarländischen Regierung eine Erklärung, derzufolge sie in allen wichtigen politischen Fragen hinsichtlich der Anwendung dieser Gesetze vor jeder Ausführungsmaßnahme die vorherige Zustimmung der Kommission einholen sollte. Hoffmann aber verweigerte sich mit dem Argument, dem Gremium werde damit ein formelles oder moralisches Vetorecht zugestanden, das aller Voraussicht nach im Anschluß an das Referendum auf den Saarkommissar

31 Vgl. Repgen, Saar-Frage, S. 106.

32 PA, BStS, Bd. 230, Vortragsnotiz Thierfelders 219-214-00/293/55, 27.6. 1955, Geheim; zu den heftigen innenpolitischen Reaktionen auf die saarländischen Gesetze s. de Margerie an Pinay, Tel. 2563/65, 1. 7. 1955, in: DDF 1955, Bd. 2, S. 1 f.

33 S. PA, BStS, Bd. 230, Thierfelder an Hallstein, Vortragsnotiz 219-214-00/298/55, 1. 7. 1955, Geheim.

34 S. Gesetz über die Durchführung der Volksbefragung, Vereinsgesetz, Versammlungsgesetz u. Pressegesetz, 8. 7. 1955, in: Schmidt, Saarpolitik, Bd. 3, S. 730-754.

35 François-Poncet an Pinay, Tel. 2654/59, 8. 7. 1955, in: DDF 1955, Bd. 2, S. 35 f., hier S. 35. 
übertragen werden müsse ${ }^{36}$. Dehousse zeigte sich einsichtig und begnügte sich mit der mündlichen Zusage Hoffmanns, er werde keine wichtigen politischen Entscheidungen treffen, ohne sich vorher mit der Kommission ins Benehmen gesetzt zu haben. Der Ministerpräsident konnte dies als wichtigen Erfolg verbuchen. Die Freude darüber wurde allenfalls dadurch getrübt, daß die Kommission von ihm verlangte, mit der Publikation der Gesetze bis nach der für den 15. 7. anberaumten nächsten Sitzung des WEU-Ministerrates zu warten. $\mathrm{Da}$ die Niederlande in diesem Gremium Einstimmigkeit für alle Saarentscheidungen verlangten, ein Einverständnis der Bundesrepublik aber nicht erwartet werden konnte, befürchtete Hoffmann eine weitere Verzögerung der Veröffentlichung ${ }^{37}$.

Seine Prophezeiung erfüllte sich, noch ehe die Tagung des Ministerrates begonnen hatte. Am 12. 7. verlangte Adenauer von Pinay, auf Hoffmann Druck auszuüben, damit die aus dem Saarland Ausgewiesenen nicht nur das Stimmrecht, sondern durch Aufhebung der Ausweisungsverfügung auch die Möglichkeit zur Rückkehr erhielten ${ }^{38}$. Einen Tag später erklärte ein Sprecher der Bundesregierung gegenüber der Presse, man habe die saarländischen Gesetzentwürfe noch nicht erhalten und könne daher auch schwerlich am 15.7. bereits Stellung nehmen ${ }^{39}$. Wollte die Bundesregierung die Durchführung des Referendums hinausschieben, da sie weiterhin von einer Mehrheit zugunsten des Statuts ausging? Als Kaiser am 9. 7. Mitglieder der Saar-CDU zur Vorbereitung ihrer Wiederzulassung nach Trier einlud, zeigte sich die Führung auf jeden Fall unschlüssig über den Ausgang und hielt ein Übergewicht der Nein-Stimmen für unwahrscheinlich ${ }^{40}$.

Von der Sorge um ein deutsch-französisches "marchandage" umgetrieben, schickte Hoffmann seinen Gesandten in Paris umgehend in den Quai d'Orsay und warnte vor jeder Verzögerung ${ }^{41}$. Pinay hielt die Vorwürfe für völlig abwegig. Ganz im Sinne des Ministerpräsidenten lehnte er jede Verschleppung der Billigung der Saargesetze ab, weil sie die Vorphase des Referendums auf den Herbst verschieben mußte, da diese erst mit dem Inkrafttreten der Gesetze beginnen konnte. Irritiert über die deutsche Halsstarrigkeit, sandte er François-Poncet sofort zu Adenauer und drohte unverhüllt mit fatalen Rückwirkungen auf die Pariser Deutschlandpolitik anläßlich der nahenden Genfer Gipfelkonferenz $z^{42}$.

Dies wollte die Bundesregierung aber nicht auf sich beruhen lassen. In persönlichen Gesprächen mit François-Poncet und de Margerie wehrten sich Adenauer und von Brentano vehement gegen die Verdächtigungen und unterstrichen, wie sehr auch sie ein Ende dieser Phase der Unsicherheit wünschten. Da sie offenbar den Beginn der deutschen Parlamentsferien abzuwarten gedachten, baten sie zur exakten Prüfung des kurz vor der Vollendung stehenden Berichtes des DehousseAusschusses um eine Verschiebung der WEU-Entscheidung um einige Tage. Eine neuerliche Sitzung des Ministerrates schien dazu nicht zwingend notwendig, da

6 S. Aufzeichnung der Unterabt. Saar im MAE, 9. 7. 1955, in: ebd., S. 42.

7 S. de Carbonnel an Pinay, Tel. 513/14, 11. 7. 1955, Réservé, in: ebd., S. 43.

8 S. PA, B 17, Bd. 22, Bl. 278f., Adenauer an Pinay, 219-214-32-20/5758/55, 12. 7. 1955, Konzept.

S. Pinay an François-Poncet, Tel. 3264/67, 13. 7. 1955, Priorité, in: DDF 1955, Bd. 2, S. 74.

to Vgl. Kosthorst, Jakob Kaiser, S. 349; Thoß, Lösung, S. $275 \mathrm{f}$.

41 Aufzeichnung Massigli, 13. 7. 1955, in: DDF 1955, Bd. 2, S. 76.

42 S. Pinay an François-Poncet, Tel. 3264/67, 13. 7. 1955, Priorité, in: ebd., S. 74; zur Genfer Deutschlandkonferenz der Vier Mächte s. Kap. IV.1.1. 
die Regierungen ihre Stellungnahme auch schriftlich an den Ständigen Rat der Westeuropäischen Union in London schicken konnten ${ }^{43}$. Sechs Tage nach der Stellungnahme der Dehousse-Kommission, die nach Sichtung der Saargesetze die für die Organisation der Volksabstimmung erforderlichen rechtlichen Voraussetzungen als erfüllt ansah ${ }^{44}$, gab die Bundesregierung in einem sehr zum Verdruß Frankreichs veröffentlichten Aide-Mémoire an die WEU zwar trotz ihrer Bedenken ebenfalls ihr Plazet. Sie legte aber Wert auf die Feststellung, daß die Saarregierung vor etwaigen Durchführungsmaßnahmen zu den Gesetzen in wichtigen politischen Fragen die Kommission um Stellungnahme bitten müsse ${ }^{45}$. Kurz darauf billigte der WEU-Rat den Bericht des Dehousse-Ausschusses; vierundzwanzig Stunden später publizierte die saarländische Regierung die vier Gesetze und setzte das Datum für die Volksabstimmung auf den 23. 10. 1955 fest $^{46}$.

\section{Ja zum Saarstatut, Nein zu Hoffmann?}

Mit diesen Entscheidungen gab Saarbrücken den Weg für die Wiederzulassung der Oppositionsparteien frei, der CDU-Saar, der Deutschen Sozialdemokratischen Partei (DSP) und der Demokratischen Partei Saar (DPS). Sechs weitere prodeutsche Parteien sollten sich in den nächsten Wochen formieren ${ }^{47}$ und trugen mit dazu bei, daß sich die Stimmung an der Saar nach diesem „Ausbruch der Freiheit" 48 schlagartig änderte. Der Kampf um das Saarstatut ließ politische Feindschaften in aller Schärfe ausbrechen, spaltete nicht nur die classe politique, sondern auch Vereine, Belegschaften und selbst Familien ${ }^{49}$. Mit Unmut beobachtete die französische Diplomatie, wie die bundesdeutsche Presse sofort den Wahlkampf eröffnete, während die französischen Zeitungen zunächst völlig desinteressiert wirkten ${ }^{50}$. Ende Juli sprachen sich DSP und DPS eindeutig für die Ablehnung des Saarstatuts aus, wobei die DSP indirekt von der SPD unterstützt wurde ${ }^{51}$. Schon nach wenigen Tagen sah sich Paris dazu genötigt, in Bonn gegen die Wahlpropaganda der neuen Organisationen zu protestieren - und lief dort offene Türen ein. Hallstein ließ in seiner Bestürzung über den von den Parteien angeschlagenen Ton gegenüber François-Poncet nichts $\mathrm{zu}$ wünschen übrig. Heinrich Schneider, der DPS-Vorsitzende, galt ihm als „forcené, Hubert Ney, der Chef der CDU-Saar als „imbécile“. Trotz der nationalistischen Auswüchse hielt er einen positiven Ausgang des Referendums indes für sicher ${ }^{52}$. Entweder

43 S. François-Poncet an Pinay, Tel. 2754/58, 14. 7. 1955, in: DDF 1955, Bd. 2, S. 76f.; ders. an dens., Tel. 2766/74, 15. 7.1955, in: ebd., S. 80-82; PA, Abg.-Liste Ministerbüro, Bd. 155, von Brentano an Adenauer, Aufzeichnung, 14. 7. 1955, Eilt sehr; ebd., B 17, Bd. 31, Bl. 100 f., van Scherpenberg an von Brentano, 410F-752-05/22-5115/55, 18. 7. 1955.

44 S. Bericht der Dehousse-Kommission, 15. 7. 1955, in: Bulletin 1955, S. $1216 \mathrm{f}$.

45 S. Aide-Mémoire der Bundesregierung, 21. 7. 1955, in: ebd., S. 1217; Interview Blüchers mit dem "Nordwestdeutschen Rundfunk", 29.7. 1955, in: ebd., S. 1192 f.; Pinay an François-Poncet, Tel. 3520/21, 30. 7. 1955, in: DDF 1955, Bd. 2, S. $159 \mathrm{f}$.

46 S. de Carbonnel an Pinay, Tel. 527/29, 23.7. 1955, in: ebd., S. 132f.; Schmidt, Saarpolitik, Bd. 3, S. 164 u. 331.

47 Vgl. ebd., S. 168-175 u. 331f.

48 Ebd., S. 331.

$49 \mathrm{Vgl}$. Hannig, Separatisten.

so S. Aufzeichnung der Unterabt. Saar im MAE, 5. 8. 1955, in: DDF 1955, Bd. 2, S. $207 \mathrm{f}$.

51 Vgl. Schmidt, Saarpolitik, Bd. 3, S. 271 f.; Thoß, Lösung, S. 276.

52 François-Poncet an Pinay, Tel. 2983/3006, 29. 7. 1955, in: DDF 1955, Bd. 2, S. 155-159, hier S. 157. 
war der Staatssekretär vom eigenen Wunschdenken gefangen oder er sagte schlicht die Unwahrheit und suchte den Botschafter in Sicherheit zu wiegen. Eine Meinungsumfrage ermittelte jedenfalls in der ersten Augustwoche ein ganz anderes Bild: $52 \%$ der Befragten sprachen sich gegen und nur noch $14 \%$ für das Statut aus ${ }^{53}$.

Vieles hing von der Haltung der CDU-Saar ab, die am 7. 8. zur Gründungsversammlung lud. Eine Ablehnung des Saarstatuts durch die Tochter der BundesCDU hielt deren Vorsitzender aus innerparteilichen wie außenpolitischen Gründen für den „größte[n] politische[n] Fehler". Einerseits betrachtete Adenauer das von ihm ausgehandelte Statut nach wie vor als „erheblichen Fortschritt an der Saar". Andererseits befürchtete er eine Spaltung innerhalb der Union. Vor allem aber hielt er es für ausgeschlossen, daß bei einem negativen Ausgang der Volksbefragung in absehbarer Zeit neue Vereinbarungen mit Frankreich getroffen werden könnten. Damit drohten „katastrophal[e]“ Rückwirkungen auf die Beziehungen zu den Westmächten und insbesondere zu Frankreich. Unmißverständlich plädierte Adenauer daher Anfang August intern gegenüber den christdemokratischen Politikern an der Saar für ein "Ja"54 und ließ dies auch über den CDU-Pressedienst verbreiten ${ }^{55}$. Doch damit nicht genug: Am 6. 8. forderte der Kanzler den rheinland-pfälzischen Ministerpräsidenten Altmeier sogar zum Fernbleiben vom Gründungsakt der CDU-Saar auf, weil ihm dessen Auftritt als Bundesratspräsident wegen des Neutralitätsgebotes außenpolitisch unerwünscht war ${ }^{56}$. Schließlich sprach er sich in einem Grußtelegramm an die Gründungsmitglieder ausdrücklich für ein positives Votum zum Saarstatut aus. Er konnte aber nicht verhindern, daß Ney die entsprechende Passage bei der Verlesung der Botschaft unterschlug und damit den beabsichtigten $Z$ weck erreichte: Einstimmig votierten die Delegierten gegen das Abkommen ${ }^{57}$.

Die emotionale Heftigkeit, mit der der Wahlkampf nun vollends entbrannte, beobachtete das Kanzleramt mit wachsender Sorge. Auf der Waagschale deutscher Interessen wogen die Solidarität der westlichen Verbündeten gegenüber dem sowjetischen Druck in Genf und die nach der Messina-Konferenz aufkeimenden Hoffnungen auf eine "relance européenne“ in den Augen Adenauers eben schwerer als die Aussicht auf ein gescheitertes Saarstatut mit ungewissen Konsequenzen. Sollten die deutschen Parteien an der Saar nicht die Mehrheit erhalten, sah er eine parteipolitische „Zersplitterung“ voraus, die nur der SPD nutzen konnte. Würde das Referendum scheitern, drohte eine Belastung des deutsch-französischen Verhältnisses mit kaum abschätzbaren Folgen für die Deutschland- und die Europapolitik. Auch die Beziehungen zu den Angelsachsen konnten wegen der von einigen Aktionen der prodeutschen Parteien wachgerufenen Reminiszenzen an die gerade einmal zehn Jahre zurückliegende braune Vergangenheit arg in Mitleiden-

53 Vgl. Repgen, Saar-Frage, S. $106 \mathrm{f}$.

54 Adenauer an Lenz, 4. 8. 1955, in: Adenauer, Briefe 1953-1955, S. 329f., hier S. 300; vgl. Schwarz, Adenauer, Bd. 2, S. 228.

55 S. Erklärung des Deutschland-Union-Dienstes, 4. 8. 1955, in: AdG 1955, S. 5298C; Schmidt, Saarpolitik, Bd. 3, S. $277 \mathrm{f}$.

$56 \mathrm{Vgl}$. Thoß, Lösung, S. 276.

57 S. Resolution der CDU Saar, 7. 8. 1955, in: Schmidt, Saarpolitik, Bd. 3, S. 219 f.; Kosthorst, Jakob Kaiser, S. 349 f.; Thoß, Lösung, S. 276. 
schaft gezogen werden ${ }^{58}$, auch wenn sie von einer Einmischung im Sinne Frankreichs nichts wissen wollten ${ }^{59}$.

Mitte August begann das Auswärtige Amt angesichts des drohenden Aufstands gegen die Hoffmann-Regierung60, sich mit dem Gedanken eines negativen Referendums auseinanderzusetzen. Zur Sprachregelung wies von Brentano seine Diplomaten in den wichtigsten europäischen Hauptstädten und in Washington an, der Auffassung Ausdruck zu verleihen, daß mit einer Ablehnung nicht zu rechnen sei. Im Fall einer gegenteiligen Entwicklung sollten sie Gespräche über die Rechtssituation vermeiden ${ }^{61}$. Intern kalkulierte die Bundesregierung die Ablehnung in ihre Überlegungen durchaus schon mit ein ${ }^{62}$. In den Reihen der Christdemokraten machte sich gar eine gewisse Genugtuung über die anschwellende prodeutsche Gefühlswelle an der Saar breit ${ }^{63}$. Da Bonn offiziell weiter auf Beruhigung der erhitzten Gemüter setzte, entstand in Paris nicht zu unrecht der Eindruck von einem deutschen „Doppelspiel“64. Wie doppelbödig die Adenauersche Saarpolitik tatsächlich war, läßt sich wohl kaum noch zweifelsfrei aufklären. Schenkt man den Memoiren von Franz Josef Strauß Glauben, stellte der Kanzler der DPS aus den „Reptilienfonds“ zehn oder elf Millionen Mark zur Verfügung65.

Überlegungen der saarländischen Regierung, der prodeutschen Agitation mit der Verhängung des Ausnahmezustandes den Nährboden zu entziehen, wurde französischerseits abgelehnt ${ }^{66}$. Auch von dem Plan einer eigenen demoskopischen Erhebung, die ein günstigeres Stimmungsbild liefern sollte, nahm man an der Seine wieder Abstand ${ }^{67}$. Statt dessen beschränkte sich Pinay darauf, Adenauer am 24. 8. schriftlich die gemeinsame Verantwortung in Erinnerung zu rufen, die beide Regierungen im vergangenen Oktober für die Saar übernommen hätten. Das wohlverstandenen Interesse beider Völker verlange nach der Ratifizierung des Saarstatuts. Geschickt setzte er den Hebel dort an, wo der Kanzler am empfindlichsten war: Ein Scheitern verursache einen vielleicht irreparablen Rückschlag für die europäische Idee68.

Die mahnenden Worte des französischen Außenministers verhallten nicht ungehört. Adenauer sah sich am 26. 8. abermals dazu veranlaßt, eine offiziöse Stellungnahme zugunsten des Saarstatuts zu verbreiten ${ }^{69}$. An der Seine wurde die Erklärung zwar begrüßt, man hielt sie aber für ungenügend. Ungeachtet der im

58 Adenauer an Altmeier, 11. 8. 1955, in: Adenauer, Briefe 1953-1955, S. 343 f., hier S. 344; s.a. Thoß, Lösung, S. 277; zur Genfer Außenministerkonferenz und „relance européenne" vgl. Kap. IV.1.1 u. IV.2.1.

59 S. Dufournier an MAE, Tel. 524/30, 29. 8. 1955, Très secret, Réservé, in: DDF 1955, Bd. 2, S. 372374; Thoß, Lösung, S. $277 \mathrm{f}$.

60 Vgl. Schmidt, Saarpolitik, Bd. 3, S. 331-342.

61 S. PA, B 17, Bd. 22, B1. 301, Grewe an Den Haag etc., Tel. 124 etc., 18. 8. 1955.

$62 \mathrm{Vgl}$. Krone, Tagebücher, Bd. 1, S. 187, Tb. 23. 8. 1955.

63 S. de Margerie an Pinay, Tel. 3291/99, 19. 8. 1955, Réservé, in: DDF 1955, Bd. 2, S. 274 f.

64 PA, Abg.-Liste Ministerbüro, Bd. 155, Unterredung zwischen Hallstein und de Margerie vom 25. 8. 1955, Vermerk; s.a. „Le Monde“, 11. 8. 1955, auch in: BDFD III, S. 503 f.

65 S. Strauß, Erinnerungen, S. 218.

66 Vgl. Hoffmann, Ziel, S. 415 f.; Hüser, Frankreich und die Saarabstimmung, S. 373.

67 S. ebd., S. 374.

68 S. Pinay an Bonn, Tel. 3715/19, 24. 8. 1955, in: DDF 1955, Bd. 2, S. $310 \mathrm{f}$.

69 S. Presseerklärung des Deutschland-Union-Dienstes, 26.8. 1955 in: AdG 1955, S. 5331 A; Schmidt, Saarpolitik, Bd. 3, S. 280-282. 
Statut von Bonn und Paris eingegangenen Verpflichtung zur Nichteinmischung bemängelte der Quai d'Orsay, daß es sich weder um eine Mitteilung des Kanzlers noch um eine der Bundesregierung handle ${ }^{70}$. Was Paris als nicht ausreichend empfand, wurde von den prodeutschen Parteien in Saarbrücken bereits als grobe Intervention gescholten. Heinrich Schneider, der Chef der DPS, forderte in der Bundesvorstandssitzung der FDP am 30. 8. die Liberalen dazu auf, weitere Eingriffe der Bonner Christdemokraten und des Kanzlers zu verhindern ${ }^{71}$.

Es mußte Adenauer zweifellos unangenehm berühren, als François-Poncet ihm am 1. 9. mit guten Wünschen Pinays für seine Moskaureise erneut mahnende Worte zur Saarfrage übermittelte und implizit in Erinnerung rief, was auf dem Spiel stand, sollte es zwischen Frankreich und der Bundesrepublik zum Bruch kommen. Die USA hatten auf der Genfer Gipfelkonferenz soeben erst ihr großes Interesse an einer Entspannung zur Sowjetunion signalisiert, während Frankreich sich als verläßlicher Partner in den deutschlandpolitischen Fragen präsentierte. Die Wiederbelebung der europäischen Einigung konnte nur dann zum Erfolg führen, wenn Deutsche und Franzosen gemeinsam die nötige Kraft aufbrachten. Was bedeutete die Saarfrage angesichts der aufschimmernden Gefahr einer außenpolitischen Vereinzelung der Bundesrepublik?

Mit der aus diesem Szenarium gezogenen Schlußfolgerung, das Saarstatut unter allen Umständen durchzuboxen, stand der Kanzler indes ziemlich allein. Zwar sorgten sich auch innerhalb der SPD einige Politiker um die Zukunft des deutschfranzösischen Verhältnisses, sie rechneten aber mit einem Nein und begrüßten ein solches Ergebnis lebhaft ${ }^{72}$. Adenauers Reaktion auf die drohende Belastung der Verbindung zu Frankreich fiel ganz anders aus. Mit bisher unbekannter Schärfe distanzierte er sich im Gespräch mit François-Poncet vom Gebaren der prodeutschen Parteien. „Il n'avait pas de termes assez durs pour stigmatiser la sottise, l'aveuglement, la brutalité des partis pro-allemands", brach es aus ihm heraus. Schneider, der ehemalige Nazi, und Ney, sie alle seien verrückt, stellten sie doch überhaupt nicht in Rechnung, was sie mit ihrer Kampagne anrichteten. Ihm selbst sei völlig klar, daß ein Scheitern des Statuts nicht zu neuen Verhandlungen führen werde. Aber er wußte auch, daß er die Prodeutschen mit seinen Tiraden nicht zum Einlenken bewegen konnte, ihnen vielmehr eine Perspektive aufzeigen mußte. Und seine Lösung hieß: Ablösung der Saarregierung. Die Opposition, versuchte er diesen Weg François-Poncet schmackhaft zu machen, sei nicht eigentlich gegen das Statut, sondern gegen den Ministerpräsidenten gerichtet. Wenn Hoffmann und Innenminister Hector verschwänden, so seine Philosophie, würde die aus Rache geborene Agitation sofort zusammenbrechen. Nach seiner Rückkehr aus Moskau wolle er auf jeden Fall seinen ganzen Einfluß geltend machen, um das Statut doch noch durchzubringen ${ }^{73}$.

70 S. de Margerie an Pinay, Tel. 3368/72, 26. 8. 1955, Réservé, in: DDF 1955, Bd. 2, S. 322f.; Tb. Blankenhorn, 28. 8. 1955, in: BDFD I, S. 486.

71 S. Sitzung des FDP-Bundesvorstandes, 30. 8. 1955, in: Wengst (Bearb.), Sitzungsprotokolle 19541960, S. 123.

72 S. François-Poncet an Pinay, Tel. 3533/38, 10. 9. 1955, Réservé, in: DDF 1955, Bd. 2, S. 468 f.; zur Haltung der SPD vgl. Cahn, Parti, Bd. 2, S. 117-120.

73 François-Poncet an Pinay, Tel. 3443/55, 2. 9. 1955, Réservé, in: DDF 1955, Bd. 2, S. 407-410, hier S. 408. 
Die Dringlichkeit des Problems erlaubte es ihm nicht, bis Mitte September zu warten. Schon einen Tag nach dem eingehenden Gedankenaustausch mit dem französischen Botschafter setzte sich der Kanzler am 2. 9. bei der Zehnjahresfeier des CDU-Landesverbandes Westfalen in Bochum erstmals auch in der Öffentlichkeit für die Annahme des Abkommens ein. Mit der Warnung vor einem drohenden Unruheherd wenige Wochen vor der Genfer Außenministerkonferenz legte er den Delegierten den seiner Ansicht nach einzig möglichen Ablauf der Dinge nahe: Anerkennung des Statuts und Wahl eines neuen Landtags, „der in seiner Mehrheit gegen die Regierung Hoffmann gerichtet ist ${ }^{\alpha 74}$.

Diese in Paris als mutig75 gewürdigte persönliche Intervention, die das Gebot der Nichteinmischung gewiß hart belastete, trug mit dazu bei, daß nun eine neue, nach außen ruhigere Phase im Abstimmungskampf begann ${ }^{76}$. Sie konnte freilich die erhitzten Gemüter nicht völlig abkühlen. Die prodeutschen Parteien schlossen sich zum „Deutschen Heimatbund“ zusammen und koordinierten das Gewicht der Neinstimmenkampagne unter der Parole „Die Saar bleibt deutsch!“77 Hinter den Kulissen reagierte die Bundesregierung mit massiven Bemühungen, Hoffmann zum Rücktritt zu bewegen ${ }^{78}$, um über den erhofften "choc psychologique" doch noch das Ruder herumzureißen und den prodeutschen Parteien das Wasser abzugraben 79 . Adenauer und von Brentano überlegten sogar mit angelsächsischen Diplomaten, ob man Pinay nicht dazu bewegen könne, der Saarbevölkerung die Rückkehr zum Status quo ante für den Fall eines negativen Votums anzudrohen ${ }^{80}$. Der französische Außenminister machte Anfang September in einem Zirkulartelegramm an seine Auslandsmissionen noch einmal unmißverständlich klar, daß es für ihn nur einen positiven Ausgang des Referendums geben dürfe. Denn ein Nein wenige Tage vor der Eröffnung der Genfer Konferenz lasse es fraglich erscheinen, so hieß es da vielsagend, ob Frankreich das von ihm bisher mitgetragene Junktim $z$ wischen Wiedervereinigung und europäischer Sicherheit weiterhin verteidigen könne. Die deutsch-französischen Beziehungen drohten in eine Sackgasse zu geraten, da Neuverhandlungen unmöglich seien ${ }^{81}$.

Je näher der Tag der Entscheidung rückte, desto gespannter blickten Bonn und Paris auf die Entwicklung in Saarbrücken. Um die innere Sicherheit in den Tagen der Volksbefragung zu gewährleisten, plädierte Pinay dafür, die von der Dehousse-Kommission empfohlene Aufstellung einer internationalen Polizei umzusetzen $^{82}$. In der Sitzung des WEU-Rates am 22. 9. sprach sich der deutsche Vertreter von Herwarth zunächst gegen solche Schutzkräfte aus. Nach einer Interven-

74 Rede Adenauers vom 2. 9. 1955 in Bochum, in: Bulletin 1955, S. 1389f., hier S. 1390; s.a. Cahn, Parti, Bd. 2, S. 68-72; Schmidt, Saarpolitik, Bd. 3, S. 282-284.

75 S. François-Poncet an Pinay, Tel. 3467/72, 3. 9. 1955, Réservé, in: DDF 1955, Bd. 2, S. $418 f$.

76 Vgl. Schmidt, Saarpolitik, Bd. 3, S. 284 f. u. 342.

7 Zitiert nach: ebd., S. 212; zur Zusammensetzung und Programmatik des Deutschen Heimatbundes s. ebd., S. 170-172, 211-252, 284 u. 351 f.; s.a. Schneider, Wunder, S. 457 f.

78 S. AMAE, Europe 1944-1960, Sarre, Bd. 276, Bl. 77 f., Konferenz der EGKS-Außenminister vom 6. 9. 1955; Hoffmann, Ziel, S. 423f.; Schmidt, Saarpolitik, Bd. 3, S. 285-290; Schwarz, Adenauer, Bd. 2, S. 229-231.

79 François-Poncet an Pinay, Nr. 1092, 9. 9. 1955, in: DDF 1955, Bd. 2, S. 467 f., hier S. 468.

$80 \mathrm{Vgl}$. Thoß, Lösung, S. 279.

81 S. Pinay an diplomatische Vertretungen, Zirkulartel. 70, 9. 9. 1955, in: DDF 1955, Bd. 2, S. $454 \mathrm{f}$.

82 S. ders. an Chauvel, Tel. 12184/87, 21.9. 1955, in: ebd., S. $536 \mathrm{f}$. 
tion von François-Poncet bei Hallstein erhielt er jedoch die Instruktion, sich dem Urteil der übrigen Delegationen anzuschließen. Nun aber stellte sich Spaak quer, und auch die übrigen Mitglieder des Rates äußerten Bedenken. Schließlich sprachen sie sich dafür aus, ihre Zustimmung vom vorherigen Einverständnis der drei beteiligten Hauptparteien Bonn, Paris und Saarbrücken abhängig zu machen und den Einsatz der Polizisten erst im Notfall zuzulassen ${ }^{83}$.

Derweil zeichnete sich aufgrund des massiven Einwirkens von Adenauer in der Bochumer Rede und seines durch die Moskau-Reise gewonnenen Prestiges eine leichte Trendverschiebung ab. In einer neuerlichen Meinungsumfrage stimmten Mitte September 19\% mit Ja, 49\% mit Nein, 23\% machten keine Angabe ${ }^{84}$. Obwohl die Beruhigung der Situation an der Saar an Rhein und an Seine sehr wohl erkannt wurde, boten die Zahlen natürlich keinen Grund zum Jubel. Im Gegenteil: Frankreich empfand die Lage an der Saar als so unbefriedigend, daß es der Bundesrepublik gegenüber andeutete, sich eventuell zu einer offiziellen Verlautbarung gezwungen zu sehen, man werde bei einem negativen Ausgang des Referendums den Status quo wiederherstellen. Da die deutschen Diplomaten dies im Gespräch mit französischen Kollegen als sehr gefährlich bewerteten, bemühte sich Pinay darum, Adenauer zu einem Treffen und einer gemeinsamen Erklärung zugunsten des Statuts zu bewegen ${ }^{85}$. Anstatt der Bitte zu folgen, schlug der Kanzler jetzt plötzlich eine völlig neue Richtung ein. Am 26. 9. unterbreitete er dem französischen Botschafter den Vorschlag, in Abänderung der im Saarstatut vorgesehenen Reihenfolge zunächst die Landtagswahlen abzuhalten und erst nach der Bildung einer neuen Regierung die Volksbefragung durchzuführen. François-Poncet konnte nicht anders, als dieses Ansinnen entschieden zurückzuweisen ${ }^{86}$.

Nachdem es Pinay gelungen war, durch Einwirkung auf den Heiligen Stuhl die offene Parteinahme des katholischen deutschen Klerus für eine Eingliederung der Saar in die Bundesrepublik zu unterbinden ${ }^{87}$, unternahm er einen neuen Anlauf, um Adenauer für ein Rendezvous zu gewinnen ${ }^{88}$. Der Kanzler mochte sich darauf aber nicht einlassen. Im Auswärtigen Amt hieß es zur Begründung, eine gemeinsame Stellungnahme über die Aufrechterhaltung des Status quo im Fall eines Scheiterns der Volksabstimmung könne kontraproduktiv wirken, weil sie mit dem Gebot der Nichteinmischung nicht in Einklang zu bringen sei und „ein bedenkliches Präjudiz für [die] allgemeine Respektierung des Saarabkommens“ darstelle eine mit Blick auf die bisherige Position Adenauers zumindest überraschende Argumentation $^{89}$. Pinay mußte einsehen, daß er mit seiner Bitte nicht durchkam. Am

83 S. MAE an Alphand, Tel. 3024/29, 24. 9. 1955, Priorité absolue, Secret, Réservé, in: ebd., S. 547549 mit Anm. 1; AMAE, Europe 1944-1960, Sarre, Bd. 276, Bl. 137, François-Poncet an MAE, Tel. 3780/81, 24. 9. 1955, Urgent.

84 Vgl. Repgen, Saar-Frage, S. 107 u. 124, Anm. 102a.

85 S. PA, B 17, Bd. 2, Bl. 188 f., von Walther an AA, FS Nr. 551, 23. 9. 1955, Verschlüsselt; FrançoisPoncet an Pinay, Tel. 3467/72, 3.9. 1955, Réservé, in: DDF 1955, Bd. 2, S. $418 \mathrm{f}$.

86 S. ders. an dens., Tel. 3799/802, 27. 9. 1955, in: ebd., S. $561 \mathrm{f}$.

87 S. Erklärung des Apostolischen Visitators für das Saarland, 27. 9. 1955, in: Schmidt, Saarpolitik, Bd. 3, S. 319; zur Parteinahme des deutschen Klerus ebd., S. 312-331; zur Démarche Pinays vgl. Pinay an London etc., Tel. 12745/51 etc., 7. 10. 1955, in: DDF 1955, Bd. 2, S. 624-626.

88 S. PA, Abg.-Liste Ref. 204, Bd. 6, von Walther an AA, FS Nr. 558, 27. 9. 1955, Verschlüsselt; ebd., B 17, Bd. 2, Bl. 204 f., ders. an dass., Tel. 562, 28. 9. 1955, Verschlüsselt.

89 Ebd., Abg.-Liste Ref. 204, Bd. 6, von Welck an Paris, Tel. 526, 28. 9. 1955, Verschlüsselt. 
28. 9. einigte er sich mit von Brentano am Rande der UNO-Vollversammlung in New York darauf, keine deutsch-französische Erklärung abzugeben. Hallstein, der dem Gespräch beiwohnte und im Gegensatz zu seinem Minister ein Scheitern des Referendums als sicher vorhersagte, brachte erneut den Gedanken vor, die Situation durch eine Demission Hoffmanns und die Verschiebung des Referendums zu retten. Pinay lehnte dies wegen der von ihm als negativ eingeschätzten Wirkung auf die französische Öffentlichkeit entschieden ab. Nur in einem Punkt herrschte zwischen den Ministern Einigkeit: dem Versuch der Familie Röchling, den im Mai vereinbarten Verkauf ihrer Firma hinauszuzögern, energisch zu widerstehen. Denn die durch den deutsch-französischen Vertrag vom 3. 5. getroffene Lösung galt beiden Seiten als die bestmögliche. Nach einigem Zögern ließ von Brentano zumindest laut französischem Protokoll schließlich auch erkennen, daß er die Aufstellung der internationalen Polizeitruppe unter der Obhut der WEU akzeptiere, sofern sie außerhalb des Saarlandes stationiert und der Dehousse-Kommission unterstellt würde ${ }^{90}$. Des weiteren willigte Adenauer noch am selben Tag in Pinays Bitte um ein Treffen ein und stimmte dem 5. 10. als Termin zu. Er bestand allerdings darauf, nicht die Saarfrage, sondern seine Moskaureise der Öffentlichkeir gegenüber als Grund anzugeben ${ }^{91}$.

Mit dem in den letzten Wochen gewonnenen Ansehen im Rücken, meinte er nun außerdem, doch ein Stück weit Pinays Wunsch nach einer erneuten Klarstellung zur Beeinflussung der Lage an der Saar entgegenkommen zu können. Als Ersatz für die gemeinsame Regierungserklärung dachte er an eine öffentliche Stellungnahme seiner Partei. Der von ihm am 30.9. einberufene Bundesvorstand zeigte ihm aber rasch die kalte Schulter. Adenauer begann mit einem seiner gewohnt langen Berichte über die außenpolitische Lage, von der seines Erachtens in geradezu Bismarckscher Diktion „alles andere abhängig“ sei92. Zur Saarfrage überleitend, betonte er die positive Wirkung des Trendumschwungs der letzten beiden Wochen. Obwohl sich die Situation an der Saar „etwas beruhigt“ habe, sei der Kampf noch keineswegs gewonnen. Eindringlich und mit wütenden Ausfällen gegen Schneider und Ney warnte er vor den fatalen Folgen eines negativen Votums, das der Bundesrepublik sowohl in Frankreich als auch in den USA erheblichen Schaden zufügen werde ${ }^{93}$.

90 S. Unterredung zwischen von Brentano und Pinay vom 28.9. 1955, Bericht, in: DDF 1955, Annexes, Bd. 2, S. $323 \mathrm{f}$. Das Dokument spricht irrtümlicherweise davon, von Brentano habe einer gemeinsamen Erklärung wohlwollend gegenübergestanden. Vgl. dagegen Alphand an MAE, Tel. 2146/49, 29. 9. 1955, in: DDF 1955, Bd. 2, S. 573 f. Bezüglich der Polizeitruppe betonte von Brentano wenige Tage später in einem Schreiben an Adenauer, er habe Pinay „dringend“ darum gebeten, den Plan „nicht weiter zu verfolgen“ (BA, NL von Brentano, Bd. 156, Bl. 56-62, von Brentano an Adenauer, 3. 10. 1955). Auch englischen Quellen zufolge fand er die Aufstellung der Polizeitruppe als zu provokativ (Thoß, Lösung, S. 280). Zur Haltung der Bundesregierung zum Vertrag über die Röchling-Werke s. Adenauer an Blücher, 6. 5. 1955, in: Adenauer, Briefe 19531955, S. 276 f.; vgl. Kap. III.3.4.

91 S. PA, B 17, Bd. 2, Bl. 213, von Welck an Paris, Tel. 527, 28. 9. 1955, Verschlüsselt.

92 Erklärung Adenauers in der Sitzung des CDU-Bundesvorstandes, 30. 9. 1955, in: Buchstab (Bearb.), Protokolle 1953-1957, S. 583-600, hier S. 584; vgl. Rede Bismarcks im Preußischen Abgeordnetenhaus, 9. 2. 1866: die auswärtigen Angelegenheiten seien „an sich Zweck" und stünden „höher als die übrigen“ (von Bismarck, Gesammelte Werke, Bd. 10, S. 264).

93 Erklärung Adenauers in der Sitzung des CDU-Bundesvorstandes, 30.9. 1955, in: Buchstab (Bearb.), Protokolle 1953-1957, S. 619-622, hier S. 619. 
Seine innerparteilichen Widersacher wie Altmeier, Hellwig und Zimmer ließen sich weder von dieser Argumentation noch von der wachsenden Empörung Adenauers beeindrucken. Aus ihrer Sicht konnte in demokratisch regierten Ländern an einer "Mehrheit von 60 bis $65 \%$ " nicht vorbeigesehen werden ${ }^{94}$. Nach zunehmend schärferen gegenseitigen Attacken mußte der Kanzler schließlich einen Kompromiß hinnehmen. Der Bundesvorstand billigte zwar noch einmal den mit dem Statut beschrittenen Weg, deklarierte dies aber weder als Aufruf an die Saarländer noch als bindende Weisung für den zum folgenden Tag einberufenen CDU-Parteiausschuß. Selbst dieser entschärften Linie wollten nur 15 von 26 Vorstandsmitgliedern folgen ${ }^{95}$. "Er hätte den Beschluß nicht fordern sollen“, mokierte sich der Fraktionsvorsitzende der Union Heinrich Krone in seinem Tagebuch. „Mehr, als von seiner Seite bisher getan wurde, sollte er nicht auf seine Schultern laden." 96

Während der Kanzler mit minimalem Erfolg bei seinen Parteifreunden für seinen Kurs kämpfte, gingen im Auswärtigen Amt neue Forderungen des Quai d'Orsay ein. Pinay beharrte darauf, daß anläßlich des bevorstehenden Treffens zwischen Adenauer und Faure in einem Kommuniqué auch die Saarfrage berührt würde 97 , und legte bereits einen Entwurf vor98. Der Kanzler wollte von einer solchen öffentlichen Verlautbarung aber nach wie vor nichts wissen und setzte, wie er dem französischen Botschafter bei dessen Abschiedsbesuch mitteilte, seine Hoffnung darauf, Druck auf die Saar-CDU ausüben zu können"9. Auch im Auswärtigen Amt betrachtete man eine öffentliche Aktion beider Regierungen mit gemischten Gefühlen. Einerseits war man der Auffassung, jede Gelegenheit zu nutzen, um die Stimmung an der Saar im Sinne des Statuts zu beeinflussen. Andererseits befürchtete man aber, das Gegenteil des Beabsichtigten zu erreichen ${ }^{100}$. Schließlich erklärte sich Adenauer doch bereit, den französischen Kommuniquéentwurf als Basis für einen deutschen Gegenvorschlag zu akzeptieren ${ }^{101}$.

Damit entfachte er aber den Unwillen seiner Kabinettskollegen. Blücher sprach sich in einem persönlichen Schreiben gegen eine deutsch-französische Erklärung aus. Auch von einer Verschiebung der Volksabstimmung hielt er nichts, da sie die Spannungen noch verlängerte. Nur ein „,freiwilliger“ Rücktritt Hoffmanns, Hektors [sic!] usw." konnte aus seiner Sicht Entlastung bringen ${ }^{102}$. Auch Kaiser und Altmeier warnten vor der zweiseitigen Deklaration, da die Saarbevölkerung ohne jede

94 Erklärung Altmeiers in der Sitzung des CDU-Bundesvorstandes, 30. 9. 1955, in: ebd., S. 627 f., hier S. 628.

95 S. Sitzung des CDU-Bundesvorstandes, 30. 9. 1955, in: ebd., S. 622-644 u. 649 f.; zum Beschluß des Bundesparteiausschusses vom 1.10. 1955 s. ebd., S. 644, Anm. 61; s.a. Repgen, Saar-Frage, S. 109112.

96 Krone, Tagebücher, Bd. 1, S. 191, Tb. 30. 9. 1955.

97 S. BA, NL Blücher, Bd. 82, Bl. 169-171, von Walther an AA, FS Nr. 567, 30. 9. 1955, Geheim, Citissime, Verschlüsselt.

98 S. ebd., Bl. 172f,, ders. an dass., FS Nr. 569, 30. 9. 1955, Streng Geheim, Citissime, Verschlüsselt.

99 S. de Margerie an Pinay, Tel. 3839/42, 1. 10. 1955, Très urgent, in: DDF 1955, Bd. 2, S. 582 f.

100 S. AMAE, Europe 1944-1960, Sarre, Bd.277, Bl. 8, ders. an MAE, Tel. 3846/51, 1. 10. 1955, Réservé.

101 S. ebd., Bl. 9, ders. an dass., Tel. 3858, 1. 10. 1955, Réservé.

102 BA, NL Blücher, Bd. 82, Bl. 165 f., Blüicher an Adenauer, AZ 3538/55, 4. 10. 1955, Rein persönlich. 
Beeinflussung von außen an die Urnen treten sollte ${ }^{103}$. Adenauer hingegen akzeptierte nun nicht nur das Regierungstreffen, sondern auch die Veröffentlichung einer gemeinsamen Verlautbarung, wobei er aber nicht nur markante Änderungen am französischen Entwurf verlangte, sondern auch eine Ausweitung der Gesprächsthemen forderte. Abgesichert durch eine positive Stellungnahme des Rechtsberaters im Auswärtigen Amt, wollte er auch die Verschiebung des Referendums, die Vorziehung der Landtagswahlen und den Rücktritt Hoffmanns ansprechen ${ }^{104}$.

Ungeachtet französischer Widerstände hielt er an diesem Plan fest. Als er Faure und Pinay am 5.10. in Luxemburg traf, unterstrich er zunächst einmal, daß die deutsch-französische Entente nach dem Referendum keinen Schaden nehmen dürfe, gleich wie das Ergebnis ausfalle ${ }^{105}$. Folgt man seinen Angaben in der Sitzung des Bundesvorstands seiner Partei vom 10.3. 1956, forderte Adenauer mit Blick auf das Verhalten der Sowjets auf der Genfer Außenministerkonferenz sodann, die Frage der Zugehörigkeit der Saar auf keinen Fall erst im Rahmen eines Friedensvertrags zu regeln, wie es das Saarstatut vorsah, sondern schon in „drei bis vier Jahren “ zu entscheiden. Weder Faure noch Pinay hätten widersprochen ${ }^{106}$. Das Protokoll weiß von dieser Gesprächspassage nichts zu berichten. Statt dessen unterrichtet es uns über den Bericht, den der Kanzler den französischen Staatsmänner über eine Unterredung mit, wie er sie nannte, sehr vernünftigen saarländischen Gewerkschaftern vom Vortage gab. Er habe ihnen klar gemacht, daß ein Scheitern des Saarstatuts die Aufrechterhaltung des Status quo bedeute. Dies hätten sie verstanden und dann angeregt, Hoffmann und Hector zum Rücktritt aufzufordern, Neuwahlen auszuschreiben und einen neuen französisch-saarländischen Wirtschaftsvertrag abzuschließen. Daß dieser Vertrag der Bundesregierung ein Dorn im Auge war, sagte Adenauer nicht. Seine Vorschläge galten Faure und Pinay aber als unannehmbar, obwohl er mehrfach insistierte. Pinay hielt zwar die Bewahrung der deutsch-französischen Freundschaft für zentral, meinte aber, es sei Aufgabe beider Regierungen, ein möglichst gutes Referendum zu erreichen. Wenige Wochen später könne das saarländische Volk die Regierung Hoffmann in den Landtagswahlen ja abwählen ${ }^{107}$.

Da sie die Meinungsverschiedenheiten nicht auszuräumen vermochten, enthielt das Kommuniqué nicht die von den Franzosen erhofften klaren Worte, sondern eine ziemlich „blasse Formel“ ${ }^{108}$. Die deutsche Presse reagierte dennoch fassungs-

103 S. Kaiser an Adenauer, 4. 10. 1955, in: Kaiser, Gewerkschafter, S. 635 f.; Altmeier an dens., 4. 10. 1955, in: Schneider, Wunder, S. 460 f.

104 S. PA, B 17, Bd. 2, Bl. 218 f., Kaufmann an dens., Stellungnahme, 30.9. 1955; Aufzeichnung de Margerie, 4. 10. 1955, in: DDF 1955, Bd. 2, S. $611 \mathrm{f}$.

105 S. Unterredung zwischen Faure, Pinay und Adenauer vom 5. 10. 1955, in: ebd., Annexes, Bd. 2, S. 362-365.

106 Erklärung Adenauers in der Sitzung des CDU-Bundesvorstandes, 10. 3. 1956, in: Buchstab (Bearb.), Protokolle 1953-1957, S. 855.

107 S. Unterredung zwischen Faure, Pinay und Adenauer vom 5. 10. 1955, in: DDF 1955, Annexes, Bd. 2, S. 357-365; MAE an London etc., Tel. 12711/19 etc., 7. 10. 1955, Urgent, Réservé, in; DDF 1955, Bd. 2, S. 626-628; Faure, Mémoires, Bd. 2, S. 516f.; Cahn, Parti, Bd. 2, S. 62-68; Thoß, Lösung, S. 280 f. Zu den deutschen Vorbehalten gegenüber dem französisch-saarländischen Wirtschaftsvertrag s. PA, B 17, Bd. 10, Bl. 201-204, Aufzeichnung Lahr 410S-300-02/Saar-3605/55, 13. 5. 1955.

108 Ebd., BStS, Bd. 229, Informationsgespräch Hallsteins, 6. 10. 1955; Kommuniqué in: Bulletin 1955, S. 1577; vgl. Schmidt, Saarpolitik, Bd. 3, S. 294-297. 
los: Was hatte Adenauer bloß bewogen, der Verlautbarung zuzustimmen? Nach den Erklärungen der Pariser Spitze müsse er doch davon ausgehen, daß Frankreich nach einer Ablehnung des Statuts den Saarländern die zugestandenen Freiheiten wieder entzöge. Für ein französischen Wohlwollen auf der Genfer Konferenz schien den Zeitungen der Preis zu hoch ${ }^{109}$. Dem Kanzler offenbar nicht, ja er legte noch eins drauf. Da die Franzosen sich mit diesem Ergebnis nicht begnügten, stimmte Adenauer einer einseitigen Presseerklärung zu, in der Pinay nach dem Treffen beteuerte, neue Verhandlungen über das Saarstatut seien weder de jure noch de facto möglich; und Faure versicherte gegenüber der amerikanischen Nachrichtenagentur CBS, das Saarstatut sei das ultimative Zugeständnis Frankreichs ${ }^{110}$.

\section{Grenzen der Staatskunst}

In einer breitgefächerten Aktion suchte Frankreich die Lage an der Saar mit weiteren Sicherungsmaßnahmen zu stabilisieren. Am 3. 10. ließ Pinay im Auswärtigen Amt ein Memorandum zur Frage der Röchling-Werke überreichen. Mit erheblichen Zusatzforderungen versuchte die Familie, den Verkauf der Werke hinauszuzögern, und nutzte dabei den Umstand, daß man bis zur Unterzeichnung der Verkaufsverträge noch immer Eigentümer war. Ihre Motive lagen auf der Hand: Erfolgte die Aufhebung der Sequester, die noch vor dem Referendum stattfinden sollte, vor der Unterzeichnung des Vertrages, wäre Röchling automatisch wieder in den Besitz der Werke gelangt. Als einziges Gegenmittel blieb Frankreich die Aufrechterhaltung der Sequester bis nach dem Referendum. Aufgrund des Bonner Widerstandes und aus Rücksichtnahme auf die psychologische Situation konzedierte Paris die Installation einer französisch-deutschen Kommission in der letzten Phase vor der Liquidation der Sequester, um die Übernahme der Werke durch die deutsch-französische Gesellschaft gemäß dem Vertrag vom 3. 5. vorzubereiten ${ }^{111}$. Als die Bundesregierung diesem Verfahren nicht zustimmte, bat Frankreich eine Woche später eindringlich darum, die Familie Röchling zum Einlenken zu bewegen ${ }^{112}$.

Zugleich richtete Pinay, der die Situation an der Saar ungeachtet der von den Parteien unter Höchstaufwand eingesetzten Propagandamittel ${ }^{113}$ mittlerweile wesentlich verbessert sah, einen lebhaften Appell an die Politiker und die Presse im Ausland, die Lage nicht zu pessimistisch zu beschreiben, sondern die Saarländer zu einem Ja zu ermutigen ${ }^{114}$. Sorgen bereitete dem Außenminister indes die Frage, wie die öffentlichen Ordnung vor und nach dem Referendum aufrechterhalten

${ }^{109}$ S. „General-Anzeiger“, 6. 10. 1955, im Auszug auch in: BDFD III, S. 505-507; Artikel Fackler, in: „Süddeutsche Zeitung“, 7. 10. 1955, im Auszug auch in: BDFD III, S. $507 \mathrm{f}$.

110 S. PA, Abg.-Liste Ref. 204, Bd. 6, von Maltzan an AA, FS Nr. 596, 7. 10. 1955, Verschlüsselt; Erklärungen Pinays und Faures in: AdG 1955, S. 5398A; MAE an London etc., Tel. 12711/19 etc., 7. 10. 1955, Urgent, Réservé, in: DDF 1955, Bd. 2, S. 626-628; Schmidt, Saarpolitik, Bd. 3, S. 292.

111 S. Couve de Murville an Pinay, Tel. 5437/46, 3. 10. 1955, in: DDF 1955, Bd. 2, S. 600-602.

112 S. PA, B 17, Bd. 3, Bl. 12, von Maltzan an AA, FS Nr. 604, 11. 10. 1955, Verschlüsselt.

113 S. Schmidt, Saarpolitik, Bd. 3, S. 353-364.

114 S. Pinay an London etc., Tel. 12745/51 etc., 7. 10. 1955, in: DDF 1955, Bd. 2, S. 624-626; PA, Abg.Liste Ref. 204, Bd. 6, von Maltzan an AA, FS Nr. 596, 7. 10. 1955, Verschlüsselt; ebd., B 17, Bd.3, Bl. 16, ders. an dass., FS Nr. 603, 11. 10. 1955, Verschlüsselt; Thoß, Lösung, S. 282. 
werden könne. Selbst wenn es zur Aufstellung der internationalen Polizei käme, was er kaum glaubte, endete deren Aufgabe mit der Verkündigung der Ergebnisse. Anschließend wäre Frankreich gemäß der Konvention vom 20.5.1953 zuständig. Für den Fall von Unruhen hatte man sich mithin so oder so auf eine Intervention eigener Truppen vorzubereiten. Daher wandte sich Pinay am 5. 10. an Verteidigungsminister Koenig und bat ihn um Mitteilung über die einzuleitenden Vorkehrungen ${ }^{115}$.

Pinays Skepsis bezüglich der Polizeitruppe war mehr als berechtigt. Den von Bonn aufgestellten Bedingungen für eine derartige Garde - Unterstellung unter den Befehl der WEU oder der Dehousse-Kommission; Stationierung außerhalb des saarländischen Territoriums; Auflösung der Truppe mit dem Tag der Bekanntgabe des Ergebnisses des Referendums - mochte Paris zwar beipflichten. Dem WEU-Rat genügte dieses Einverständnis aber nicht, weil ihm die Einwilligung Saarbrückens fehlte ${ }^{116}$. Ungeachtet der Intervention de Carbonnels weigerte sich Hoffmann aber hartnäckig, die Formierung der internationalen Polizei offiziell zu akzeptieren. Allenfalls offiziös war er bereit, entsprechende Vorbereitungen hinzunehmen und für den Fall einer Intervention eine Sondersitzung des Landtags in Aussicht zu stellen, um die gesetzliche Basis zu schaffen ${ }^{117}$. Wenngleich weder Bonn noch Paris oder die Dehousse-Kommission dieses Procedere guthießen, blieb der WEU-Rat dabei, nicht über den Kopf der saarländischen Politiker hinweg zu entscheiden. Am 10.10. beschloß er, mit den technischen Schritten zur Aufstellung zu beginnen und sich durch einen Vertreter von Dehousse über die politische Lage kurz vor der Volksbefragung informieren zu lassen ${ }^{18}$. In hektischen Sitzungen stritt der Rat in den Tagen danach um das Datum der Bekanntmachung der Rekrutierung und den Termin der Aufstellung selbst. Zu einer Einigung kam es nicht, weil die sieben Vertreter nicht der von Sorge vor Unruhen gekennzeichneten Empfehlung Frankreichs und der Bundesrepublik folgen wollten, die Polizeitruppe möglichst geheim zu halten ${ }^{119}$. Als England mit der Forderung aufwartete, man müsse mit dem spätestmöglichen Datum der Entsendung der nationalen Truppenteile am 17.10. auch der Bekanntgabe der Aufstellung zustimmen, zog Frankreich sein Gesuch ganz zurück, weil man die an der Saar eingekehrte Ruhe nicht gefährden wollte ${ }^{120}$. Im Gegenzug ließ die Regierung kurz vor der Abstimmung an der Grenze zwischen Lothringen und der Saar die eigene „Garde mobile“ zusammenziehen, um für den Notfall gewappnet zu sein ${ }^{121}$.

115 S. AMAE, Europe 1944-1960, Sarre, Bd. 277, BI. 49-51, Pinay an Koenig, Nr. 741, 5. 10. 1955, Minute.

116 S. Chauvel an Pinay, Tel. 4369/78, 6. 10. 1955, Urgent, Réservé, in: DDF 1955, Bd. 2, S. 619-621.

117 S. de Carbonnel an dens., Tel. 708/13, 8. 10. 1955, Réservé, in: ebd., S. 633-635.

118 S. Chauvel an dens., Tel. 4450/58, 11. 10. 1955, Très urgent, Réservé, in: ebd., S. 639-641; de Carbonnel an dens., Tel. 721/24, 12. 10. 1955, Priorité, Réservé, in: ebd., S. $651 \mathrm{f}$.

119 S. Chauvel an dens., Tel. 4506/12, 12. 10. 1955, Priorité absolue, Réservé, in: ebd., S. 652f.; Pinay an Chauvel, Tel. 12898/902, 13. 10. 1955, Priorité absolue, Réservé, in: ebd., S. 654 f.; Chauvel an Massigli, Tel. 4530, 14. 10. 1955, Très secret, Priorité la plus absolue, Réservé, in: ebd., S. 664; ders. an Pinay, Tel. 4540/42, 14. 10. 1955, Priorité absolue, Réservé, in: ebd., S. 664 f.

120 S. Pinay an Chauvel, Tel. 13040/42, 16. 10. 1955, Priorité absolue, Réservé, nebst Aide-mémoire, in: ebd., S. 680 f.; ders. an dens., Tel. 13056/59, 17. 10. 1955, Priorité, Réservé, in: ebd., S. 681 f.; Thoß, Lösung, S. 283.

121 Vgl. Schmidt, Saarpolitik, Bd. 3, S. 377. 
Auch Adenauer setzte jetzt ganz auf Kalmierung. Als die Bonner Oppositionsparteien in diesen Tagen einen Antrag ins Parlament einbrachten, mit dem sie die Bundesregierung aufforderten, rechtzeitig Maßnahmen zu treffen, um beim Scheitern des Saarstatuts Schwierigkeiten für die Saar auf wirtschaftlichem und sozialem Gebiet zu vermeiden, lehnte die Union die Behandlung kurzerhand ab ${ }^{122}$. Nach einem neuerlichen Vorstoß der Gegner des Statuts, welche Gutachten juristischer Kapazitäten veröffentlichten, aus denen hervorging, daß eine Ablehnung nicht zum Status quo zurückführen dürfe ${ }^{123}$, schickte der Kanzler Staatssekretär Globke mit einer Reihe von Vorschlägen in die französische Botschaft, die beweisen sollten, daß ihm tatsächlich an einer Billigung des Saarabkommens gelegen $\operatorname{sei}^{124}$. Das Pariser Kabinett erneuerte daraufhin am 19. 10. in einer Verlautbarung seinen Standpunkt, Neuverhandlungen kämen nach einem Nein nicht in Frage ${ }^{125}$. Einen Tag später bekräftigte Pinay diese Ansicht noch einmal vor dem Auswärtigen Ausschuß der Assemblée Nationale ${ }^{126}$. Absprachegemäß ${ }^{127}$ begrüßte Adenauer diese umgehend veröffentlichte „letzte Warnung“ an die Saarländer ${ }^{128}$ und betonte in einem Interview am 20. 10. erneut: „Der Weg zu einer anderen Regierung an der Saar zu kommen, geht über die Anerkennung des Statuts und die Wahl eines Landesparlaments, das in seiner Zusammensetzung gegen die Regierung Hoffmann gerichtet ist." 129

Wenngleich der Kanzler sich also so weit, wie es ihm innenpolitisch als Regierungschef möglich erschien, für ein Ja einsetzte, zog das Auswärtige Amt auch den Fall eines Scheiterns des Referendums in sein Kalkül mit ein. Nach den letzten Meldungen aus Paris gab es keinen Anlaß daran zu zweifeln, daß Frankreich sich kaum zu Lösungen durchringen würde, die keine Rückkehr zum Status quo bedeuteten. Thierfelder sprach intern gar die Besorgnis aus, Paris werde bei einem Nein zum Saarstatut „die Saar selbst wieder in die Hand nehmen, vielleicht sogar notfalls Truppen in das Saargebiet entsenden" und den WEU-Rat vor einer Einflußnahme ausschalten. Von Brentano hielt das zwar für ausgeschlossen, erteilte aber am 21.10. den Auftrag, ein Memorandum vorzubereiten, um notfalls am

122 Vgl. ebd., S. 273.

${ }^{123}$ S. Erklärung des Deutschen Saarbundes, 17. 10. 1955, in: Dischler, Saarland, Bd. 1, S. 125 f.; de Margerie an Pinay, Tel. 4014/20, 18. 10. 1955, in: DDF 1955, Bd. 2, S. 688 f.; Schmidt, Saarpolitik, Bd. 3, S. 275 f.

124 S. AMAE, Europe 1944-1960, Sarre, Bd. 277, Bl. 219-221, de Margerie an MAE, Tel. 4002/13, 18. 10. 1955, Réservé, Priorité; ebd., Crouy-Chanel an Bonn, Tel. 4305/08, 18. 10. 1955, Réservé, Priorité; ebd., Bl. 240, de Margerie an MAE, Tel. 4054/55, 19. 10. 1955, Réservé, Priorité; ders. an Pinay, Tel. 4036/46, 19. 10. 1955, Priorité absolue, Réservé, in: DDF 1955, Bd. 2, S. 694-697.

125 Vgl. Schmidt, Saarpolitik, Bd. 3, S. 293.

126 S. Erklärung Pinays vor dem Auswärtigen Ausschuß der Assemblée Nationale, 20.10. 1955, in: Dischler, Saarland, Bd. 1, S. 126 f.; Hüser, Frankreich und die Abstimmung, S. 377; Schmidt, Saarpolitik, Bd. 3, S. 293.

127 S. PA, B 17, Bd. 3, Bl. 45 f., von Maltzan an AA, FS Nr. 625, 20. 10. 1955, Verschlüsselt; de Margerie an Pinay, Tel. 4036/46, 19. 10. 1955, Priorité absolue, Réservé, in: DDF 1955, Bd. 2, S. 694-697; Schumacher, Konrad Adenauer, S. 73.

128 So äußerte sich Carstens gegenüber dem Schweizer Vertreter in Bonn (Rebsamen an Eidgenössisches Departement für Auswärtige Angelegenheiten, 20.10. 1955, in: Todt ( $\mathrm{Hg}$.), Anfangsjahre, S. 176).

129 Interview Adenauers mit „dpa“, 20. 10. 1955, in: AdG 1955, S. 5422; s.a. Dischler, Saarland, Bd. 1, S. $127 \mathrm{f}$. 
24. 10. den Ratsmitgliedern in Paris den deutschen Standpunkt mitzuteilen ${ }^{130}$. Adenauer wies ihn am Tag darauf schriftlich an, sich in bezug auf neue Verhandlungen über die Saarfrage „nicht in einen Gegensatz zu Frankreich“ zu stellen, sei doch in der Vergangenheit von deutscher Seite soviel geschehen, was Beanstandungen des Partner rechtfertige ${ }^{131}$. Zugleich ließ der Kanzler beim französischen Außenminister anfragen, ob beide Regierungen nicht eine Geste vorbereiten könnten, um eine etwaige gefährliche Entwicklung an der Saar sofort zu bereinigen. Er dachte dabei an einen Telegrammaustausch mit dem Président du Conseil, eventuell auch zwischen den beiden Außenministern, der den Vorteil hätte, der Öffentlichkeit gegenüber als spontaner Akt der Regierungschefs präsentiert zu werden. Paris wollte davon aber noch nichts wissen, sondern die Auszählung der Stimmen abwarten ${ }^{132}$.

Aus einem nicht gezeichneten französischen Bericht geht hervor, daß man im Quai d'Orsay zwar gute Chancen für ein Ja sah, sich aber ein breites Handlungsmuster für das Nein zurechtlegte. Sollte das Referendum scheitern, galt die Fortsetzung der Regierung Hoffmann als „la plus plausible“, die Abhaltung der Landtagswahlen hingegen als unmöglich, weil sie die Mehrheitsverhältnisse zweifellos ändern würde. Der ungenannte Autor war sogar bereit, Aufruhr und Generalstreik hinzunehmen, und glaubte, sie durch den Einsatz der Streitkräfte wie die Ablösung konzilianter Minister durch solche wie Hector bekämpfen zu können. Sollte das Gewaltszenario trotz des Verbleibs von Hoffmann ausbleiben, hielt man es im Quai d'Orsay offenbar für möglich, Hector fallenzulassen, um Hoffmann zu halten. „En tout état de cause, le maintien du Président Hoffmann au pouvoir entraine le recours à la force sauf si, contrairement à nos affirmations répétées, nous acceptons de négocier à nouveau avec l'Allemagne." Allerdings wurde ein Rücktritt Hoffmanns nicht völlig ausgeschlossen, zumindest theoretisch in seinen Konsequenzen durchdekliniert, wobei der dabei ausgearbeitete Plan recht genau dem entsprach, was sich in dieser Angelegenheit tatsächlich entwickeln sollte. Zwei Möglichkeiten schienen in diesem Fall gegeben: sofortige Demission oder Abdankung nach den Landtagswahlen. Gingen sie, was zu erwarten war, im Sinne der Deutschen aus, mußte man von den prodeutschen Parteien und vor allem der CDU-Saar als der wichtigsten und am wenigstens scharfen Kraft die Zusage erhalten, daß die Zoll- und Wirtschaftsunion für eine gegebene Zeit aufrechterhalten bliebe ${ }^{133}$.

Ungeachtet der wiederholten deutschen und französischen Plädoyers zugunsten des Saarstatuts, dem sich unmittelbar vor der Öffnung der Wahllokale sehr zum Verdruß des Deutschen Heimatbundes auch noch Dehousse in einer Rundfunkrede anschloß ${ }^{134}$, sprachen sich die Saarländer am 23. 10. nach einem überaus heftigen Wahlkampf zu 67,7\% gegen das Statut aus. 32,3\% stimmten mit Ja. Die Wahlbeteiligung lag bei $96,7 \%{ }^{135}$. Wie erklärt sich dieses „Wunder an der Saar" ${ }^{136}$ ?

130 PA, BStS, Bd. 230, Thierfelder an Hallstein, Vortragsnotiz, 21. 10. 1955.

131 Adenauer an von Brentano, 22. 10. 1955, in: Adenauer, Briefe 1955-1957, S. 76-78, hier S. 76.

132 S. de Margerie an Pinay, Tel. 4096/100, 22. 10. 1955, Priorité absolue, Réservé, in: DDF 1955, Bd. 2, S. $705 \mathrm{f}$.

133 AMAE, Europe 1944-1960, Sarre, Bd. 277, Bl. 267-271, Aufzeichnung, (20.) 10. 1955.

134 Vgl. Schmidt, Saarpolitik, Bd. 3, S. 301-304.

135 S. ebd., S. 364-371; AdG 1955, S. 5422. 
Die politisch-ökonomische Sogwirkung durch die Bundesrepublik, die sich zu einer starken Konkurrenz entwickelnde lothringische Stahlindustrie, die sich abzeichnende sinkende Nachfrage nach saarländischer Kohle in Frankreich und die sich daraus ergebende Notwendigkeit zur Erschließung neuer Märkte, vor allem in Süddeutschland, aber auch der französische Kampf um die Röchling-Werke sowie die kaum demokratisch zu bezeichnenden Verhältnisse an der Saar hatten in der Bevölkerung einen Stimmungsumschwung bewirkt ${ }^{137}$, den die prodeutschen Parteien in ihrem Kampf gegen die „Hoffmann-Demokratur" sofort zu nutzen wußten.

Obwohl die Urnen noch bis 18 Uhr geöffnet waren, gestand Pinay gegenüber seinem Vertreter an der Saar schon in der Mittagszeit die Niederlage Frankreichs ein. Ausdrücklich bekannte er, den Idealen der europäischen Kooperation treubleiben zu wollen, machte aber zugleich klar, daß jetzt nur die Bewahrung des Status quo in Frage käme: „il n'y a d'autre possibilité raisonnable que le maintien de la situation actuellement existante “138. Dieser Marschroute entzog die Regierung Hoffmann noch in der Nacht in gewisser Weise den Boden, indem sie nach der Bekanntgabe des vorläufigen Endergebnisses kurz nach null Uhr - wohl nicht ganz freiwillig - ihren Rücktritt erklärte ${ }^{139}$.

Hatten Faure und Pinay eine Chance versäumt, stärker in den Wahlkampf einzugreifen, wie Grandval kritisierte ${ }^{140}$, ohne zu bedenken, daß dann Adenauer angesichts der beiderseitigen Pflicht zur Neutralität kaum seinen Kurs hätte aufrechterhalten können? Hatten sie die Sache schleifenlassen, weil die Lösung der Saarfrage „nie als Primärziel, sondern höchstens als Sekundärziel im Rahmen weitergehender außenpolitischer Konzeptionen “ galt ${ }^{141}$ ? Trifft der daraus schimmernde Vorwurf der Fahrlässigkeit wirklich den Kern der Sache angesichts des Zwangs, vor dem Hintergrund des EVG-Debakels und des Algerienkonfliktes auf die Öffentlichkeit in Westeuropa Rücksicht nehmen zu müssen?

Im Auswärtigen Amt weigerten sich die Beamten, „lange Gesichter [zu] machen, wie es offenbar der Bundeskanzler tut“. Sie freuten sich, daß Adenauer ein Jahr zuvor mit seinem Wunsch, statt eines Referendums nur einen Landtagsentscheid durchzuführen, bei Mendès France auf Granit gestoßen war ${ }^{142}$. Der Kanzler betrachtete das Ergebnis als schwere politische Schlappe, wenngleich er später glauben zu machen versuchte, daß er die Ablehnung des Saarstatuts erhofft habe ${ }^{143}$. Weder diese Selbststilisierung zum "vorausschauenden Übertaktiker“" noch die Klage seiner Gegner, der Kanzler sei zur Preisgabe der Saar bereit gewe-

136 Schneider, Wunder.

137 S. Lahr an seinen Bruder, 24. 10. 1955, in: Lahr, Zeuge, S. 236-238; Schwarz, Ära Adenauer 19491957, S. 282.

138 Pinay an de Carbonnel, Tel. 1061/64, 23. 10. 1955, Priorité absolue, Réservé, Diffusion restreinte, in: DDF 1955, Bd. 2, S. 728.

139 S. Erklärung der saarländischen Regierung, 23./24. 10. 1955, in: EA 1955, S. 8391; Hoffmann, Ziel, S. 426; Schmidt, Saarpolitik, Bd. 3, S. 371-374.

$140 \mathrm{Vgl.} \mathrm{Hüser,} \mathrm{Frankreich} \mathrm{und} \mathrm{die} \mathrm{Abstimmung,} \mathrm{S.} 379$.

141 Ebd., S. 378.

142 Lahr an seinen Bruder, 24. 10. 1955, in: Lahr, Zeuge, S. 236.

143 S. Aufzeichnung Adenauer, 24, 10. 1955, in: Adenauer, Briefe 1955-1957, S. 80f.; Adenauer, Erinnerungen 1953-1955, S. 377. 
sen, entspricht allerdings der Wirklichkeit ${ }^{144}$. Nicht die Zielvorgabe, sondern die Frage nach der Rangordnung des Saarproblems im größeren außenpolitischen Konnex unterschied Adenauer von seinen Kritikern. Im Oktober 1955 wünschte er sich zweifelsohne ein Ja zum Saarstatut; aber ebenso gewiß wollte er das Provisorium einer zeitweilig autonomen Saar rechtlich offenhalten. Adenauer dachte nicht daran, dieses deutsche Gebiet abzuschreiben ${ }^{145}$.

Nachdem die Saarländer einen Strich durch seine Rechnung gemacht hatten, ging es ihm vor allem um Schadenbegrenzung. Noch am späten Abend des 23. 10. gab das Auswärtige Amt zur Sprachregelung an die Auslandsmissionen die Parole aus, man respektiere die neu geschaffene Situation, setze aber alles daran, die deutsch-französische Zusammenarbeit und die Bemühungen um die europäische Integration nicht zu beschädigen ${ }^{146}$. Vom Krankenlager aus sandte Adenauer nach Bekanntwerden des vorläufigen Endergebnisses am folgenden Tag ein persönliches Telegramm an Faure, in dem er ebenfalls seiner festen Überzeugung Ausdruck gab, daß der Ausgang der Volksabstimmung die guten Beziehungen zwischen beiden Staaten "nicht beeinträchtigen darf und wird“"147.

Genau dies wurde in der französischen Presse nicht unbedingt so gesehen. Es überwogen zwar die maßvollen Kommentare, unter denen man sogar solch bemerkenswerte Stimmen wie die von Albert Finet fand, der vollstes Verständnis für die Saarländer äußerte: „Si j'étais Sarrois, j'aurais voté non.“148 Es gab aber auch anderslautende Auffassungen, etwa des linken "Combat", der am Tag nach der Abstimmung die Prognose stellte, die „polémique franco-allemande va s'ouvrir plus ardente que jamais" 149 .

Um einen derartigen Konflikt zu verhindern, stellte die französische Regierung noch am selben Tag in einem Kommuniqué fest, sie nehme das enttäuschende Ergebnis zur Kenntnis, wolle aber dem Ideal der europäischen Zusammenarbeit treubleiben. Diplomatisch verklausuliert hieß es dann weiter: „Die dadurch geschaffene Lage kann nur im legalen Rahmen der bestehenden Einrichtungen und Strukturen betrachtet werden." 150 Wollte sich Paris also offiziell noch nicht über den nunmehr einzuschlagenden Kurs äußern, so ließ doch ein Briefaustausch zwischen Adenauer und Pinay erkennen, wie sehr beide Seiten auf eine einvernehmliche Lösung bedacht waren, ohne die von den Angelsachsen bereits intern abgestimmte Hilfe von außen ${ }^{151}$ in Anspruch nehmen zu wollen. Natürlich hatten die Saarländer den Deutschen und Franzosen ein großes Hindernis in den Weg gelegt. Doch mochten sich die Nachbarn dadurch nicht vom eingeschlagenen Weg der engen Entente abbringen lassen. "Maintenant plus que jamais", so rief der Außenminister dem Kanzler zu, „nous devons redoubler d'efforts pour éliminer les causes de discorde dans le respect des intérêts essentiels de chacun et pour atteindre

144 Thoß, Lösung, S. 285.

145 Vgl. ebd., S. $285 f$.

146 S. PA, B 17, Bd. 3, Bl. 68f., von Welck an Paris etc., Tel. 599 etc., 23. 10. 1955, Verschlüsselt, Citissime.

147 Adenauer an Faure, Tel., 24. 10. 1955, in: Bulletin 1955, S. 1679; s.a. Cahn, Second retour, S. 15 f.

148 Artikel Finet, in: „Réforme“, 29. 10. 1955, im Auszug auch in: BDFD III, S. 514-516, hier S. 516.

${ }_{149}$ Artikel Fabiani, in: „Combat“, 24. 10. 1955, im Auszug auch in: BDFD III, S. 508 f., hier S. 509.

150 Kommuniqué des Quai d’Orsay, 24. 10. 1955 (Übersetzung), in: EA 1955, S. 8391.

151 Vgl. Thoß, Lösung, S. 281-283. 
les objectifs que nous nous sommes fixés." Wesentlich warmherziger, als es Faure in einem Telegramm an Adenauer ausdrückte, unterzeichnete er sein Schreiben mit "mes sentiments les plus amicaux"152. Die Bundesregierung empfand diese Haltung keineswegs als selbstverständlich. Als von Brentano am Nachmittag dieses 24. 10. mit Pinay in Paris zur letzten Abstimmung für die Genfer Außenministerkonferenz zusammentraf ${ }^{153}$, tat er es mit unverkennbarem Unbehagen, konnte sich aber rasch persönlich davon überzeugen, wie unbegründet seine Sorgen waren ${ }^{154}$. War Frankreich, so stellte sich nun die alles entscheidende Frage, aber bereit, von seiner bisher stets betonten Linie abzuweichen und in neue Verhandlungen mit der Bundesrepublik über die Saarfrage einzutreten?

152 Pinay an Adenauer, mitgeteilt in: ders. an François-Poncet, Tel. 4378, 25. 10. 1955, Priorité, in: DDF 1955, Bd. 2, S. 735; s.a. PA, BStS, Bd. 350, Adenauer an Pinay, 24. 10. 1955, in französischer Übersetzung in: DDF 1955, Bd. 2, S. 735; vgl. Faure an Adenauer, 24. 10. 1955, in deutscher Übersetzung in: Bulletin 1955, S. 1679.

153 Vgl. Kap. IV.1.1.

154 Vgl. PA, BStS, Bd. 338, Erklärung von Brentanos auf der Konferenz der Missionschefs in Bonn, 8. 12. 1955, Abschrift der Tonbandaufnahme; ebd., Konferenz der Missionschefs vom 8. 12. 1955, Geheim. 


\section{IV.3.2 Der Luxemburger Vertrag vom 27. 10.1956}

„Difficile, sinon impossible, d'envisager un retour pur et simple au statu quo“

Das Saarstatut war gescheitert, aber eine Ersatzlösung nicht in Sicht. Die Regierungen in Bonn und Paris bemühten sich um Kalmierung, ohne zu wissen wie es nun weitergehen sollte. Vieles, wenn nicht alles, kam jetzt auf die Reaktion Frankreichs an, die freilich zum Gutteil davon abhing, wie die Deutschen mit dem Ergebnis des Saarreferendums umgingen. Häme, Schadenfreude oder gar nationalistische Aufwallungen waren gewiß kontraproduktiv. Die Anhänger der deutschfranzösischen Verständigung beobachteten mit Erleichterung, wie bescheiden, ja zurückhaltend das Gefühl des Triumphes in Bonn und andernorts ausfiel. Zufriedenheit ja, aber selbst bei den Befürwortern der prodeutschen Saarfraktion keine nationalistische Explosion, meldete der französische Geschäftsträger de Margerie am Abend nach der Volksabstimmung über die Atmosphäre in Bonn. Allerdings kam er nicht umhin zu betonen, daß ein Gedanke sämtliche Kommentare durchzöge: Die Rückkehr zum Status quo sei nunmehr unmöglich. Auch die Bundesregierung, dessen war er überzeugt, werde zweifellos Neuverhandlungen fordern, ohne die auf Frankreich zukommenden Schwierigkeiten zu unterschätzen1. In diesem Sinne konnte auch ein Kommuniqué des Bundeskabinetts interpretiert werden, das nach ihrer Sitzung vom 24. 10. veröffentlicht wurde. Mit dem Nein zum Saarstatut, so hieß es da, stelle sich „,von neuem die Aufgabe“ eine Lösung zu finden, die dem Willen der Saarländer Rechnung trage und sowohl der deutschfranzösischen Zusammenarbeit als auch dem Ziel der europäischen Gemeinschaft diene $^{2}$.

Obwohl London und Washington sich für den nun eingetretenen Notfall intern darauf verständigt hatten, der Rückkehr zum Status quo ante im Grundsatz Rechnung zu tragen ${ }^{3}$, ging man in Paris davon aus, mit der Ankündigung einer solchen Lösung an der Saar Schwierigkeiten heraufzubeschwören und den Protest der Partner in der WEU zu provozieren. André François-Poncet, der ehemalige französische Botschafter, hielt es in einem Artikel des „Figaro" nach dem „vote néfaste" $z$ war für logisch und juristisch gerechtfertigt, zum Status quo ante zurückzukehren. Er fügte aber sofort einschränkend hinzu, man könne den Ausgang des Referendums nicht einfach ignorieren ${ }^{4}$. Eine Resolution der SFIO bedauerte zwar ausdrücklich das Votum im Saarland, lehnte aber die Aufrechterhaltung des IstZustandes als schlechteste aller Politiken ab ${ }^{5}$. Ähnlich argumentierte auch der Quai d'Orsay. Dort hieß es am Tag nach dem Plebiszit, es sei "difficile, sinon impossible, d'envisager un retour pur et simple au statu quo". Da das Referendum ein Votum gegen die Autonomie der Saar bedeute, sei Bonn nun der einzige echte

1 S. de Margerie an Pinay, Tel. 4106/20, 24. 10. 1955, Urgent, Réservé, in: DDF 1955, Bd. 2, S. 729 731; vgl. Artikel Dombrowski, in: „Frankfurter Allgemeine Zeitung“, 26. 10. 1955, im Auszug auch in: BDFD III, S. 512-514.

2 Kommuniqué der Bundesregierung, 24. 10. 1955, in: Bulletin 1955, S. 1679; s.a. PA, B 17, Bd. 3, B1. 73, von Welck an Washington etc., Tel. 770 etc., 24. 10. 1955, Citissime.

3 Vgl. Thoß, Lösung, S. 281.

4 Artikel François-Poncet, in: „Le Figaro“, 25. 10. 1955, im Auszug auch in: BDFD III, S. 510-512, hier S. 510.

5 S. Resolution des Parteivorstands der SFIO, 25. 10. 1955, in: ebd., S. $509 \mathrm{f}$. 